\title{
Identification of risk factors for in-hospital death of COVID - 19 pneumonia -- lessions from the early outbreak
}

\author{
Zhigang Wang ${ }^{1}$ and Zhiqiang Wang ${ }^{2^{*}}$ (D)
}

\begin{abstract}
Background: To examine the clinical characteristics and identify independent risk factors for in-hospital mortality of 2019 novel coronavirus (COVID-19) pneumonia.

Methods: A total of 156 patients diagnosed with COVID-19 pneumonia at the Central Hospital of Wuhan from January 29, 2020, to March 20, 2020, and 20 healthy individuals were enrolled in this single-centered retrospective study. The epidemiological parameters, clinical presentations, underlying diseases, laboratory test results, and disease outcomes were collected and analyzed.

Results: The median age of all enrolled patients was 66 years. At least one underlying disease was identified in 101 COVID-19 patients, with hypertension being the most common one, followed by cardiovascular disease and diabetes. The most common symptoms identified upon admission were fever, cough, dyspnea, and fatigue. Compared to survival cases, patients who died during hospitalization had higher plasma levels of D-dimer, creatinine, creatine kinase, lactate dehydrogenase, lactate, and lower percentage of lymphocytes (LYM [\%]), platelet count and albumin levels. Most enrolled patients received antibiotics and anti-viral treatment. In addition, 60 patients received corticosteroids, and 51 received intravenous immunoglobulin infusion. Forty-four patients received noninvasive ventilation and 19 received invasive ventilation. Respiratory failure was the most frequently observed complication (106 [67.9\%]), followed by sepsis (103 [66.0\%]), acute respiratory distress syndrome (ARDS) (67 [42.9\%]), and septic shock (50 [32.1\%]).

Multivariable regression suggested that advanced age (OR [odds ratio] $=1.098,95 \% \mathrm{Cl}$ [confidence interval]: 1.0061.199, $P=0.037)$, shorter duration from onset to admission ( $\mathrm{OR}=0.853,95 \% \mathrm{Cl}: 0.750-0.969, P=0.015)$ and elevated lactate level upon admission ( $\mathrm{OR}=2.689,95 \% \mathrm{Cl}: 1.044-6.926, P=0.040)$ were independent risk factors for inhospital mortality for COVID-19 infection. Meanwhile, increased LYM (\%) at admission (OR = 0.787, 95\% Cl: 0.686$0.903, P=0.001)$ indicated a better prognosis.
\end{abstract}

Conclusions: In this study, we discovered that age, duration from onset to admission, LYM (\%), and lactate level upon admission were independent factors that affecting the in-hospital mortality rate.

Keywords: Characteristics, COVID-19, Risk factors, Therapy, Survivors

\footnotetext{
* Correspondence: medsciwang@yahoo.com

${ }^{2}$ Department of Obstetrics and Gynecology, The Central Hospital of Wuhan, Wuhan 430014, China

Full list of author information is available at the end of the article
}

(c) The Author(s). 2021 Open Access This article is licensed under a Creative Commons Attribution 4.0 International License, which permits use, sharing, adaptation, distribution and reproduction in any medium or format, as long as you give appropriate credit to the original author(s) and the source, provide a link to the Creative Commons licence, and indicate if changes were made. The images or other third party material in this article are included in the article's Creative Commons licence, unless indicated otherwise in a credit line to the material. If material is not included in the article's Creative Commons licence and your intended use is not permitted by statutory regulation or exceeds the permitted use, you will need to obtain permission directly from the copyright holder. To view a copy of this licence, visit http://creativecommons.org/licenses/by/4.0/ The Creative Commons Public Domain Dedication waiver (http://creativecommons.org/publicdomain/zero/1.0/) applies to the data made available in this article, unless otherwise stated in a credit line to the data. 


\section{Background}

Since it was first identified, the 2019 novel coronavirus (COVID-19) has relentlessly spread all over the world and infected almost 42 million people as of Oct 23, 2020, and taken more than 1.1 million lives [1, 2]. The COVID-19 is the seventh member of the coronavirus family [3]. Compared with the $10 \%$ fatal rate in SARS$\mathrm{CoV}$ infection [4] and 37\% in MERS-CoV infection [5], the mortality rate of COVID-19 seems to be lower and has been estimated around 1-5\% [2]. However, COVID19 has a higher reproduction number (RO) therefore is much more contagious than its precursors, which results in an enormous burden to global health. The clinical symptoms of COVID-19 infection are generally mild, and most patients have a good prognosis. However, the conditions can deteriorate in about $10-20 \%$ of all patients who are often required to be transferred to intensive care unit (ICU) and suffered from a very high mortality rate [6].

Due to its latent onset, it is important to early identify patients with increased risks of disease progression so clinical treatment can be adjusted before disease progression. Even though numerous reports have been published about COVID-19, studies that focus on identifying such risk factors are still needed. Here in this retrospective analysis, we identified several risk factors that associated with death in COVID-19 patients and assessed the effectiveness of current treatment strategies.

\section{Methods}

A total of 156 patients diagnosed with COVID-19 at the Central Hospital of Wuhan from January 29, 2020, to March 20, 2020, and 20 healthy individuals (age/sex matched) were included in this single-centered retrospective study. Epidemiology parameters, clinical presentations, laboratory results, and disease outcomes of enrolled patients were collected and reviewed for COVID-19 patients by two independent designated researchers. While in the control group, only laboratory results were collected.

The diagnosis was made following the Chinese COVID19 management guideline (7th Edition) [7], and the disease severity was characterized as mild, moderate, severe, or critical according to the same guideline. More specifically, the diagnosis for the suspected patients required confirmational real-time PCR tests for COVID-19 nucleic acid with samples obtained from patients' throat swabs and/or bronchoalveolar lavage fluid (BALF).

For severe and critical patients, corticosteroid therapy (methylprednisolone 40-120 mg per day) was given immediately upon admission or within the first 3 days of hospitalization. Antibiotics and Oseltamivir were empirically applied to all patients. Oxygen support (nasal cannula, high oxygen flow, noninvasive assisted ventilation, and mechanical ventilation) was applied to patients as needed.

\section{Definitions}

Fever was defined as an axillary temperature above $37.3^{\circ} \mathrm{C}$. Sepsis and septic shock were defined according to the 2016 Third International Consensus Definition for Sepsis and Septic Shock [8]. Acute respiratory distress syndrome (ARDS) was diagnosed according to the Berlin Definition [9]. Acute kidney injury was identified according to the Kidney Disease: Improving Global Outcomes definition (KDIGO) guideline [10]. The acute cardiac injury was diagnosed if serum levels of cardiac enzymes increased above the upper limit of the normal value or new abnormalities identified in electrocardiography and echocardiography [6].

Discharge criteria was defined as: body temperature returned to normal and maintained at least three consecutive days; remission of respiratory symptoms; significant improvement on chest computed tomography $(\mathrm{CT})$ scans and negative results on RNA tests on nasopharyngeal swabs obtained at least $24 \mathrm{~h}$ apart.

\section{Statistical analysis}

Data was analyzed with SPSS software for windows (version 25.0 IBM Corp, Armonk, NY). Continuous variables were expressed as median and interquartile (IQR). The student $t$-test was applied for normally distributed continuous variables and the Mann-Whitney $U$ test for nonnormally distributed variables. Categorical variables were compared with the $x^{2}$ test or Fisher exact test.

All parameters that might affect in-hospital mortality were screened by univariable analyses. The variables that reached significance were further tested by multivariable stepwise logistic regression analyses (stepwise enter method). After the risk factors were determined, KaplanMeier survival curves were plotted to reveal the effect of laboratory risk factors on in-hospital mortality. Receiveroperating characteristic (ROC) curves were constructed to assess the diagnostic value of the laboratory test results. The optimal cutoff was first assessed by Youden's index $(J=$ Sensitivity + Specificity -1$)$. STATA statistical analysis software was used to assess the difference between the areas under the receiver-operating characteristic curve (AUC). For laboratory results, we considered the normal ranges used in the Central Hospital of Wuhan as the reference. $P<0.05$ was considered statistically significant.

\section{Results}

The cohort included in this study consisted of 156 hospitalized patients diagnosed with COVID-19 pneumonia and 20 healthy controls. Among all patients, 56 died during hospitalization and 100 were successfully 
discharged. The median age of all enrolled patients was 66 years (IQR, 46.3-73.0; range, 9-99 years), and 76 (48.7\%) were male. Comorbidities were identified in 101 (64.7\%) patients, with hypertension, cardiovascular disease, and diabetes being the most common ones. The most common symptoms presented upon admission were fever (79 [50.6\%]) and cough (71 [45.5\%]), followed by dyspnea (28 [17.9\%]) and fatigue (23 [14.7\%]) (Table 1).

Compared with successfully discharged patients, the patients who died during hospitalization were older (72.0 years [65.3-83.0] VS 58.0 years [37.0-69.0]), and were more likely complicated with underlying diseases such as hypertension (34 [60.7\%] vs 31 [31.0\%]), diabetes $(20$ [35.7\%] vs 11 [11.0\%]), cardiovascular disease (27 [48.2\%] vs 17 [17.0\%]), cerebrovascular disease (13 [23.2\%] vs 9 [9.0\%]), chronic obstructive pulmonary disease (COPD) (15 [26.8\%] vs 12 [12.0\%]), cancer (11 [19.6\%] vs 3 [3.0\%]), chronic renal disease (11 [19.6\%] vs $4[4.0 \%])$, and chronic liver disease $(12[21.4 \%]$ vs 2
[2.0\%]). Compared with survivors, non-survivors were more likely to present with fatigue $(15$ [26.8\%] vs 8 [8.0\%]), anorexia (9 [16.1\%] vs $4[4.0 \%])$, and neuropsychic symptoms (4 [7.1\%] vs 0$)$ (Table 1$)$.

In terms of laboratory tests, multiple differences between survivors and non-survivors were identified and summarized in Table 2. Specifically, non-survivors had an increased level of white blood cell (WBC) count, neutrophil count, percentage of neutrophils, D-dimer, creatinine, creatine kinase (CK), and lactate dehydrogenase (LDH), as well as higher levels of c-reactive protein (CRP), procalcitonin (PCT) and interleukin-6 (IL-6). Whereas, percentage of lymphocytes, platelet count, and albumin levels were significantly lower in non-survivors. In addition, non-survivors had elevated levels of lactate and glucose, accompanied by lower levels of $\mathrm{PaO}_{2} / \mathrm{FiO}_{2}$ (Table 3). Furthermore, our result suggested that the level of LYM (\%) in COVID-19 patients upon admission was significantly lower than that in the control group. In

Table 1 Baseline characteristics of patients infected with COVID-19

\begin{tabular}{|c|c|c|c|c|}
\hline & $\begin{array}{l}\text { Total } \\
(n=156)\end{array}$ & $\begin{array}{l}\text { Survivor } \\
(n=100)\end{array}$ & $\begin{array}{l}\text { Non-survivor } \\
(n=56)\end{array}$ & $P$ value $^{\mathrm{a}}$ \\
\hline \multicolumn{5}{|l|}{ Characteristics } \\
\hline Age, years & $66.0(46.3-73.0)$ & $58.0(37.0-69.0)$ & $72.0(65.3-83.0)$ & $<0.001$ \\
\hline Male, \% & $76(48.7)$ & $44(44.0)$ & $32(57.1)$ & 0.115 \\
\hline \multicolumn{5}{|l|}{ Underlying disease } \\
\hline Cardiovascular disease, \% & $44(28.2)$ & $17(17.0)$ & $27(48.2)$ & $<0.001$ \\
\hline COPD, \% & $27(17.3)$ & $12(12.0)$ & $15(26.8)$ & 0.019 \\
\hline Chronic renal disease, \% & $15(9.6)$ & $4(4.0)$ & $11(19.6)$ & 0.001 \\
\hline Chronic liver disease, \% & $14(9.0)$ & $2(2.0)$ & $12(21.4)$ & $<0.001$ \\
\hline Cerebrovascular, \% & $22(14.1)$ & $9(9.0)$ & $13(23.2)$ & 0.014 \\
\hline Diabetes, \% & $31(19.9)$ & $11(11.0)$ & $20(35.7)$ & $<0.001$ \\
\hline Hypertension, \% & $65(41.7)$ & $31(31.0)$ & $34(60.7)$ & $<0.001$ \\
\hline Cancer, \% & $14(9.0)$ & $3(3.0)$ & $11(19.6)$ & $<0.001$ \\
\hline \multicolumn{5}{|l|}{ Initial symptoms } \\
\hline Fever, \% & 79 (50.6) & $56(56.0)$ & $23(41.4)$ & 0.074 \\
\hline Cough, \% & $71(45.5)$ & $45(45.0)$ & $26(46.4)$ & 0.864 \\
\hline Chest tightness, \% & $28(17.9)$ & $16(16.0)$ & $12(21.4)$ & 0.397 \\
\hline Asthma, \% & $30(19.2)$ & $22(22.0)$ & $8(14.3)$ & 0.241 \\
\hline Headache, \% & $5(3.2)$ & $2(2.0)$ & $3(5.4)$ & 0.351 \\
\hline Myalgia, \% & $2(1.3)$ & $1(1.0)$ & $1(1.8)$ & 1.000 \\
\hline Chill, \% & $4(2.6)$ & $4(4.0)$ & $0(0)$ & 0.297 \\
\hline Nausea or vomiting, \% & $8(5.1)$ & $3(3.0)$ & $5(8.9)$ & 0.136 \\
\hline Fatigue, \% & $23(14.7)$ & $8(8.0)$ & $15(26.8)$ & 0.002 \\
\hline Diarrhea, \% & $2(1.3)$ & $1(1.0)$ & $1(1.8)$ & 0.359 \\
\hline Poor appetite, \% & $13(8.3)$ & $4(4.0)$ & $9(16.1)$ & 0.014 \\
\hline Disturbance of consciousness, \% & $4(2.6)$ & $0(0)$ & $4(7.1)$ & 0.015 \\
\hline
\end{tabular}

Notes: Data presented as n (\%) or median (IQR)

Abbreviations: COVID-19 Coronavirus Disease 2019, IQR interquartile range, COPD Chronic obstructive pulmonary disease

${ }^{a} P$ values indicate differences between survivors and non-survivors. $P<0.05$ was considered statistically significant 
Table 2 Laboratory findings of patients infected with COVID-19 on admission to hospital

\begin{tabular}{|c|c|c|c|c|}
\hline & $\begin{array}{l}\text { Total } \\
(n=156)\end{array}$ & $\begin{array}{l}\text { Survivor } \\
(n=100)\end{array}$ & $\begin{array}{l}\text { Non-survivor } \\
(n=56)\end{array}$ & $P$ value $^{\mathrm{a}}$ \\
\hline WBC count, $10^{9} / \mathrm{L}$ & $6.2(4.7-8.3)$ & $5.6(4.5-7.2)$ & $7.8(5.5-12.6)$ & $<0.001$ \\
\hline Neutrophil count, $10^{9} / \mathrm{L}$ & $4.0(3.0-6.8)$ & $3.4(2.5-5.0)$ & $6.8(4.5-11.3)$ & $<0.001$ \\
\hline Lymphocyte count, $10^{9} / \mathrm{L}$ & $1.2(0.7-1.7)$ & $1.4(1.0-1.9)$ & $0.7(0.5-1.1)$ & 0.503 \\
\hline NEU (\%), \% & $71.0(58.9-84.4)$ & $62.9(55.3-72.4)$ & $86.7(76.3-91.0)$ & $<0.001$ \\
\hline LYM (\%), \% & $19.7(10.5-30.6)$ & $27.6(18.4-33.5)$ & $8.7(4.7-14.3)$ & $<0.001$ \\
\hline Hemoglobin, g/L & 123.7 (114.2-136.6) & $125.0(115.5-136.3)$ & $122.4(113.9-135.8)$ & 0.246 \\
\hline Platelet, $10^{9} / \mathrm{L}$ & $194.0(157.0-249.0)$ & $218.5(172.3-259.5)$ & $168.0(114.0-200.0)$ & $<0.001$ \\
\hline Total bilirubin, mmol/L & $10.9(7.5-17.2)$ & $10.7(7.5-14.6)$ & $13.0(7.5-25.6)$ & 0.057 \\
\hline $\mathrm{LDH}, \mathrm{U} / \mathrm{L}$ & $197.0(159.5-279.0)$ & $175.0(149.0-219.0)$ & 310.5 (201.0-479.3) & $<0.001$ \\
\hline$A L T, U / L$ & $20.2(13.5-39.5)$ & $19.5(13.0-37.9)$ & $22.1(14.7-41.5)$ & 0.400 \\
\hline AST, U/L & $21.7(16.1-34.2)$ & $18.8(15.1-26.6)$ & $30.0(21.3-55.3)$ & 0.002 \\
\hline Albumin, g/L & $37.5(33.8-42.6)$ & $39.9(36.5-43.3)$ & $33.1(29.7-33.6)$ & $<0.001$ \\
\hline Globulin, g/L & $28.7(24.4-32.9)$ & $28.2(24.1-30.9)$ & $31.5(24.8-34.9)$ & 0.014 \\
\hline $\mathrm{BUN}, \mathrm{mmol} / \mathrm{L}$ & $4.7(3.7-6.2)$ & $4.2(3.3-5.3)$ & $6.2(5.0-10.8)$ & $<0.001$ \\
\hline Creatinine, $\mu \mathrm{mol} / \mathrm{L}$ & $66.1(50.3-84.2)$ & $64.8(50.9-75.1)$ & $74.2(47.0-126.9)$ & 0.008 \\
\hline $\mathrm{CK}, \mathrm{U} / \mathrm{L}$ & $68.0(45.0-121.0)$ & $63.0(40.0-96.0)$ & $112.8(62.3-245.0)$ & 0.028 \\
\hline CK-MB, U/L & $1.6(0.8-4.6)$ & $0.9(0.7-1.4)$ & $4.3(1.7-13.3)$ & 0.044 \\
\hline troponin I, pg/ml & $20.0(4.1-57.5)$ & $10.0(3.0-20.0)$ & $50.0(22.3-115.0)$ & 0.013 \\
\hline BNP, ng/L & $118.5(32.5-392.7)$ & $56.8(20.0-132.9)$ & $374.7(135.1-814.5)$ & 0.069 \\
\hline D-dimer, mg/L & $1.0(0.4-4.6)$ & $0.7(0.2-1.6)$ & $3.3(1.2-7.8)$ & 0.025 \\
\hline CRP, mg/L & $0.9(0.1-3.6)$ & $0.2(0.1-1.6)$ & $4.1(2.5-7.2)$ & $<0.001$ \\
\hline Procalcitonin, ng/mL & $0.06(0.04-0.14)$ & $0.05(0.04-0.06)$ & $0.37(0.12-0.77)$ & 0.022 \\
\hline IL-6, pg/mL & $7.1(2.4-24.7)$ & $2.9(1.5-7.4)$ & $79.6(9.6-212.5)$ & 0.027 \\
\hline CD19+, count/ $\mu \mathrm{L}$ & $12.6(9.0-18.9)$ & $11.1(8.6-17.0)$ & $16.6(10.1-19.1)$ & 0.292 \\
\hline CD3+, count $/ \mu \mathrm{L}$ & $68.9(57.4-75.3)$ & $70.6(62.0-76.7)$ & $66.8(54.6-71.4)$ & 0.329 \\
\hline CD4+, count/ $\mu \mathrm{L}$ & $38.8(34.6-46.0)$ & $38.8(31.4-45.2)$ & $39.4(36.2-52.5)$ & 0.183 \\
\hline CD8+, count/ $\mu \mathrm{L}$ & $25.3(19.2-32.3)$ & $26.7(19.6-33.9)$ & $20.5(13.9-30.5)$ & 0.063 \\
\hline CD4/CD8 & $1.6(1.1-2.3)$ & $1.5(1.0-2.1)$ & $1.8(1.2-3.9)$ & 0.071 \\
\hline
\end{tabular}

Notes: Data presented as $\mathrm{n}(\%)$ or median (IQR)

Abbreviations: COVID-19 Coronavirus Disease 2019, WBC White blood cell, NEU (\%) Percentage of neutrophils, LYM (\%) Percentage of lymphocytes, LDH Lactate dehydrogenase, ALT Alanine aminotransferase, AST Aspartate aminotransferase, BUN Blood urea nitrogen, CK Creatine kinase-MB, CK-MB Creatine kinase-MB, BNP Brain natriuretic peptide, CRP C-reactive protein, IL-6,Interleukin-6

${ }^{a} P$ values indicate differences between survivors and non-survivors. $P<0.05$ was considered statistically significant

contrast, the levels of CRP, D-dimer, and lactate were higher in COVID-19 patients (Table 4).

$150(96.2 \%)$ patients received antibiotics and 139 (89.1\%) received antiviral treatment. Unsurprisingly, systematic corticosteroid was more commonly applied in non-survivors. Fifty-six patients $(35.9 \%)$ received high-flow nasal cannula oxygen therapy, 44 (28.2\%) received non-invasive mechanical ventilation and 19 patients $(12.2 \%)$ required invasive mechanical ventilation support. Six patients (3.8\%) received renal replacement therapy (RRT) and no patients were treated with extracorporeal membrane oxygenation therapy. Oxygen support (including high oxygen flow, noninvasive assisted ventilation, and mechanical ventilation) and renal replacement therapy was more commonly applied in non-survivors (Table 5).

The clinical outcomes of the enrolled 156 COVID-19 patients were summarized in Table 6. Unsurprisingly, non-survivor patients were more likely to develop complications compared with survivors. Respiratory failure was the most frequently developed complication (106 [67.9\%]), followed by sepsis (103 [66.0\%]), ARDS (67 [42.9\%]), septic shock (50 [32.1\%]), arrhythmia (42 [26.9\%]), acute cardiac injury (26 [16.7\%]), cardiac failure (24 [15.4\%]), and acute kidney injury (18 [11.5\%]). The median time from disease onset to admission was 10.0 days (IQR 4.3-16.0). And the median time from disease onset to discharge was 36.0 days (IQR 27.3-48.0), 
Table 3 Blood gas analysis of patients infected with COVID-19

\begin{tabular}{|c|c|c|c|c|}
\hline & $\begin{array}{l}\text { Total } \\
(n=156)\end{array}$ & $\begin{array}{l}\text { Survivor } \\
(n=100)\end{array}$ & $\begin{array}{l}\text { Non-survivor } \\
(n=56)\end{array}$ & $P$ value $^{a}$ \\
\hline $\mathrm{Ph}$ & 7.44 (7.39-7.47) & $7.43(7.40-7.46)$ & 7.45 (7.39-7.48) & 0.970 \\
\hline $\mathrm{PaO}_{2}, \mathrm{~mm} \mathrm{Hg}$ & $91.0(64.3-119.0)$ & $95.0(79.0-129.0)$ & $72.0(50.0-116.0)$ & 0.049 \\
\hline $\mathrm{PaO}_{2} / \mathrm{FiO}_{2}, \mathrm{~mm} \mathrm{Hg}$ & $195.0(90.0-262.5)$ & $225.0(152.5-287.5)$ & $117.5(78.3-192.9)$ & $<0.001$ \\
\hline $\mathrm{PaCO}_{2}, \mathrm{~mm} \mathrm{Hg}$ & $38.0(34.0-42.0)$ & $40.0(35.0-45.0)$ & $36.0(33.0-40.0)$ & 0.113 \\
\hline $\mathrm{BE}, \mathrm{mmol} / \mathrm{L}$ & $1.9(-0.7-4.1)$ & $2.1(-0.1-3.9)$ & $1.2(-1.8-4.6)$ & 0.300 \\
\hline $\mathrm{K}^{+}, \mathrm{mmol} / \mathrm{L}$ & $3.8(3.4-4.1)$ & $3.8(3.5-4.2)$ & $3.8(3.3-4.1)$ & 0.768 \\
\hline $\mathrm{Na}^{+}, \mathrm{mmol} / \mathrm{L}$ & $140.0(136.0-143.8)$ & $141.0(138.0-144.0)$ & $137.0(133.0-142.0)$ & 0.641 \\
\hline $\mathrm{Ca}^{2+}, \mathrm{mmol} / \mathrm{L}$ & $0.9(0.7-1.0)$ & $0.9(0.7-1.1)$ & $0.9(0.7-1.0)$ & 0.482 \\
\hline Lactate, mmol/L & $1.7(1.2-2.3)$ & $1.5(1.1-2.1)$ & $2.0(1.5-2.9)$ & 0.005 \\
\hline Hematocrit, \% & $37.0(32.0-41.0)$ & $38.0(32.5-42.0)$ & $36.0(30.0-40.0)$ & 0.627 \\
\hline Glucose, mmol/L & $6.7(5.4-8.7)$ & $5.9(4.9-7.9)$ & $7.4(6.2-11.1)$ & 0.034 \\
\hline
\end{tabular}

Notes: Data presented as median (IQR)

Abbreviations: COVID-19 Coronavirus Disease 2019, $\mathrm{PaO}_{2}$ Partial pressure of oxygen, $\mathrm{PaO}_{2}$ Partial pressure of carbon dioxide, $\mathrm{BE}$ Base excess

${ }^{a} P$ values indicate differences between survivors and non-survivors. $P<0.05$ was considered statistically significant

whereas the median time to death was 17.0 days (IQR 10.0-26.5). After comparing the time from disease onset to hospital admission and total hospital stay between non-survivors with survivors [(median time, 6.0 days [1.0-10.0] vs 14.5 days [7.0-20.0]) and (median time, 9.0 days [3.3-16.0] vs 22.0 days [16.0-29.0]) respectively], it seemed like the disease progressed more rapidly in nonsurvivors.

Next, multivariable logistic regression assay discovered that age (OR [odds ratio] $=1.098,95 \% \mathrm{CI}$ [confidence interval]: $1.006-1.199, P=0.037)$, duration from onset to admission $(\mathrm{OR}=0.853,95 \% \mathrm{CI}: 0.750-0.969, P=0.015)$, LYM (\%) at admission (OR $=0.787,95 \%$ CI: $0.686-$ $0.903, P=0.001)$, and lactate at admission $(\mathrm{OR}=2.689$, 95\% CI: 1.044-6.926, $P=0.040$ ) were independent risk factors for in-hospital death of COVID-19 pneumonia (Table 7).

The Kaplan-Meier survival curve showed a trend toward poorer survival in COVID-19 patients with increased lactate levels and/or decreased LYM (\%) upon admission $(P=0.020$ and $P<0.001$, respectively) (Fig. 1). In addition, we conducted the ROC curve assay and calculated the AUC among the following three indicators. We discovered that the AUC of LYM (\%) was 0.903 (95\% CI, 0.856-0.949), 0.792 (95\% CI, $0.720-0.863)$ for $\mathrm{D}$-dimer and of 0.651 (95\% CI, 0.555-0.748) for lactate (Fig. 2). Comparing to the other indicators, the AUC of LYM (\%) was higher in predicting in-hospital death (LYM [\%] VS D-dimer, $P=0.003$; LYM [\%] VS lactate, $P<0.001$; respectively). Furthermore, we discovered that the cutoff value of LYM (\%) for predicting in-hospital death was $14.7 \%$.

\section{Discussion}

In this study, we reported a cohort of 156 patients with laboratory-confirmed COVID-19 pneumonia. After carefully summarized and compared patients' clinical characteristics, we identified several risk factors for in-hospital death. Specifically, our data suggested that advanced age, shorter duration from onset to admission, decreased

Table 4 Comparision of the laboratory levels between the COVID-19 and healthy control group

\begin{tabular}{llll}
\hline & Covid-19 group $(\boldsymbol{n}=\mathbf{1 5 6})$ & Control group $(\boldsymbol{n}=\mathbf{2 0})$ & $\boldsymbol{P}$ value \\
\hline Age, years & $66.0(46.3-73.0)$ & $66.0(44.8-73.3)$ & 0.968 \\
Male, \% & $76(48.7)$ & $10(50)$ & 0.886 \\
WBC count, $10^{9} / \mathrm{L}$ & $6.2(4.7-8.3)$ & $5.6(4.0-7.0)$ & 0.090 \\
LYM (\%), \% & $19.7(10.5-30.6)$ & $27.2(22.2-34.1)$ & $\mathbf{0 . 0 0 3}$ \\
Creatinine, $\mu \mathrm{mol} / \mathrm{L}$ & $66.1(50.3-84.2)$ & $67.8(53.5-75.0)$ & 0.891 \\
CRP, $\mathrm{mg} / \mathrm{L}$ & $0.9(0.1-3.6)$ & $0.4(0.1-0.6)$ & $\mathbf{0 . 0 1 6}$ \\
D-dimer, $\mathrm{mg} / \mathrm{L}$ & $1.0(0.4-4.6)$ & $0.4(0.2-0.7)$ & $<\mathbf{0 . 0 0 1}$ \\
Lactate, $\mathrm{mmol} / \mathrm{L}$ & $1.7(1.2-2.3)$ & $1.2(0.8-1.4)$ & $<\mathbf{0 . 0 0 1}$ \\
\hline
\end{tabular}

Notes: Data presented as $\mathrm{n}(\%)$ or median (IQR)

Abbreviations: COVID-19 Coronavirus Disease 2019, WBC White blood cell, LYM (\%) Percentage of lymphocytes, CRP C-reactive protein

$P<0.05$ was considered statistically significant 
Table 5 Treatments of patients infected with COVID-19

\begin{tabular}{|c|c|c|c|c|}
\hline & $\begin{array}{l}\text { Total } \\
(n=156)\end{array}$ & $\begin{array}{l}\text { Survivor } \\
(n=100)\end{array}$ & $\begin{array}{l}\text { Non-survivor } \\
(n=56)\end{array}$ & $P$ value $^{\mathrm{a}}$ \\
\hline Antibiotics, \% & $150(96.2)$ & $96(96.0)$ & $54(96.4)$ & 1.000 \\
\hline Antiviral treatment, $\%$ & $139(89.1)$ & $89(89.0)$ & $50(89.3)$ & 0.956 \\
\hline Corticosteroids, \% & $60(38.5)$ & $22(22.0)$ & $38(67.9)$ & $<0.001$ \\
\hline Intravenous immunoglobulin, \% & $51(32.7)$ & $18(18.0)$ & $33(58.9)$ & $<0.001$ \\
\hline High-flow nasal cannula oxygen therapy, \% & $56(35.9)$ & $26(26.0)$ & $30(53.6)$ & 0.001 \\
\hline Non-invasive mechanical ventilation, $\%$ & $44(28.2)$ & $13(13.0)$ & $31(55.4)$ & $<0.001$ \\
\hline Invasive mechanical ventilation, $\%$ & $19(12.2)$ & $1(1.0)$ & $18(32.1)$ & $<0.001$ \\
\hline RRT, \% & $6(3.8)$ & $1(1.0)$ & $5(8.9)$ & 0.023 \\
\hline
\end{tabular}

Notes: Data presented as $\mathrm{n}(\%)$

Abbreviations: COVID-19 Coronavirus Disease 2019, RRT Renal replacement therapy

${ }^{\text {a }} P$ values indicate differences between survivors and non-survivors. $P<0.05$ was considered statistically significant

LYM (\%) and increased lactate at admission were associated with higher odds of in-hospital death.

The Central Hospital of Wuhan is the largest tertiary hospital around the Huanan Seafood Wholesale Market area, were speculated to be ground zero for this pandemic [11-13]. Considering the fact that most of the enrolled patients lived close to this market, our data might include a large portion of patients with so-called "primary infection". Comparing with other reports $[6,14$, 15], patients included in our cohort were older and more commonly complicated with underlying diseases. It seemed like that the patients in our study were much severer when they were diagnosed and had a longer hospital stay.

Our data suggested symptoms like fatigue, anorexia, and neuropsychic presentations were more common in the critically ill patients. The onset and persistence of these symptoms might suggest an unfavorable prognosis.
Time from disease onset to admission and death was much shorter for non-survivors, which might imply a more rapid disease progression.

After carefully reviewed the medical records of all enrolled patients, we found that $26.9 \%$ of patients with COVID-19 pneumonia presented with arrhythmia and $16.7 \%$ complicated with acute cardiac injuries. Further analysis indicated that the incidence of myocardial injury was much higher in non-survivors. In Li's report [16], they found at least $8 \%$ of patients with COVID-19 suffered an acute cardiac injury and this ratio was $12 \%$ in Wang's research [15]. The pathogenesis of COVID-19 infection-related acute myocardial injury is still understudied. But according to the clinical presentation and available laboratory results, we speculated that the direct assault from the virus, hypoxemia induced by pneumonia, and over-reacting immune response all play important roles in the pathogenesis.

Table 6 Outcomes of patients infected with COVID-19

\begin{tabular}{|c|c|c|c|c|}
\hline & $\begin{array}{l}\text { Total } \\
(n=156)\end{array}$ & $\begin{array}{l}\text { Survivor } \\
(n=100)\end{array}$ & $\begin{array}{l}\text { Non-survivor } \\
(n=56)\end{array}$ & $P$ value $^{a}$ \\
\hline Arrhythmia, \% & $42(26.9)$ & $13(13.0)$ & $29(51.8)$ & $<0.001$ \\
\hline Sepsis, \% & $103(66.0)$ & $49(49.0)$ & $54(96.4)$ & $<0.001$ \\
\hline ARDS, $\%$ & $67(42.9)$ & $17(17.0)$ & $50(89.3)$ & $<0.001$ \\
\hline Respiratory failure, $\%$ & $106(67.9)$ & $50(50.0)$ & $56(100)$ & $<0.001$ \\
\hline Cardiac failure, $\%$ & $24(15.4)$ & $11(11.0)$ & $13(35.9)$ & 0.043 \\
\hline Septic shock, \% & $50(32.1)$ & $9(9.0)$ & $41(73.2)$ & $<0.001$ \\
\hline Acute kidney injury, \% & $23(14.7)$ & $8(8.0)$ & $15(26.8)$ & 0.002 \\
\hline Acute cardiac injury, \% & $26(16.7)$ & $6(6.0)$ & $20(35.7)$ & $<0.001$ \\
\hline Onset to admission, days & $10.0(4.3-16.0)$ & $14.5(7.0-20.0)$ & $6.0(1.0-10.0)$ & $<0.001$ \\
\hline Hospitalization, days & $18.0(11.0-27.8)$ & $22.0(16.0-29.0)$ & $9.0(3.3-16.0)$ & $<0.001$ \\
\hline Onset to discharge or death, days & $30.0(21.0-42.8)$ & $36.0(27.3-48.0)$ & $17.0(10.0-26.5)$ & $<0.001$ \\
\hline
\end{tabular}

Notes: Data presented as $\mathrm{n}(\%)$ or median (IQR)

Abbreviations: COVID-19 Coronavirus Disease 2019, ARDS Acute respiratory distress syndrome

${ }^{a} P$ values indicate differences between survivors and non-survivors. $P<0.05$ was considered statistically significant 
Table 7 Risk factors associated with in-hospital death infected with COVID-19

\begin{tabular}{|c|c|c|c|c|c|c|}
\hline & Univa & alyses & & Multive & alyses & \\
\hline & OR & $95 \% \mathrm{Cl}$ & $P$ value & OR & $95 \% \mathrm{Cl}$ & $P$ value \\
\hline Age & 1.083 & $1.051-1.117$ & $<0.001$ & 1.098 & $1.006-1.199$ & 0.037 \\
\hline Onset to admission & 0.870 & $0.821-0.922$ & $<0.001$ & 0.853 & $0.750-0.969$ & 0.015 \\
\hline Cardiovascular disease & 4.546 & $2.170-9.523$ & $<0.001$ & 0.321 & $0.239-12.863$ & 0.581 \\
\hline Hypertension & 3.440 & $1.737-6.814$ & $<0.001$ & 0.124 & $0.012-1.278$ & 0.079 \\
\hline Diabetes & 4.495 & $1.957-10.322$ & $<0.001$ & 2.744 & $0.323-23.304$ & 0.355 \\
\hline Creatinine & 1.012 & $1.004-1.021$ & 0.005 & 1.006 & $0.997-1.015$ & 0.171 \\
\hline CRP & 1.427 & $1.223-1.665$ & $<0.001$ & 1.086 & $0.897-1.313$ & 0.398 \\
\hline LYM (\%) & 0.816 & $0.765-0.871$ & $<0.001$ & 0.787 & $0.686-0.903$ & 0.001 \\
\hline D-dimer & 1.047 & $1.001-1.095$ & 0.047 & 0.987 & $0.941-1.036$ & 0.599 \\
\hline Lactate & 1.738 & $1.209-2.498$ & 0.003 & 2.689 & $1.044-6.926$ & 0.040 \\
\hline Corticosteroids & 7.485 & $3.594-15.590$ & $<0.001$ & 1.162 & $0.889-1.518$ & 0.064 \\
\hline Immunoglobulin & 6.536 & $3.127-13.663$ & $<0.001$ & 2.896 & $0.771-10.877$ & 0.115 \\
\hline Acute kidney injury & 4.207 & $1.654-10.703$ & 0.003 & 12.502 & $0.188-832.413$ & 0.238 \\
\hline Acute cardiac injury & 8.704 & $3.234-23.421$ & $<0.001$ & 14.875 & $0.536-187.673$ & 0.123 \\
\hline Cardiac failure & 2.446 & $1.013-5.907$ & 0.047 & 1.788 & $0.075-42.669$ & 0.720 \\
\hline
\end{tabular}

Abbreviations: COVID-19 Coronavirus Disease 2019, OR Odds ratio, Cl Confidence interval, CRP C-reactive protein, LYM (\%) Percentage of lymphocytes

$P<0.05$ was considered statistically significant

Consistently, our study confirmed that advanced age was associated with increased mortality in patients with COVID-19, as reported by Zhou and colleagues [14]. Previously, advanced age has been identified as an important predictor of mortality in SARS and MERS infection [17, 18]. Though has not been verified in mechanism studies, several reasons may contribute to this age-related vulnerability: firstly, patients with advanced ages are usually suffered decreased cardiopulmonary compliance and reserve thus more difficulty in coping with the disequilibrium of the cardiopulmonary system induced by COVID-19 infection; secondly, previous studies indicated that advance age was associated with more robust host innate responses but decreased in cellular as well as humoral immune functions during virus infection $[19,20]$; thirdly, aged patients have an increased risk of having comorbidities which have been proved in several studies associate with worse prognosis; finally, the diagnosis and treatments in patients with advanced ages are more likely to be delayed due to atypical symptoms. Thus, attention should be paid to COVID-
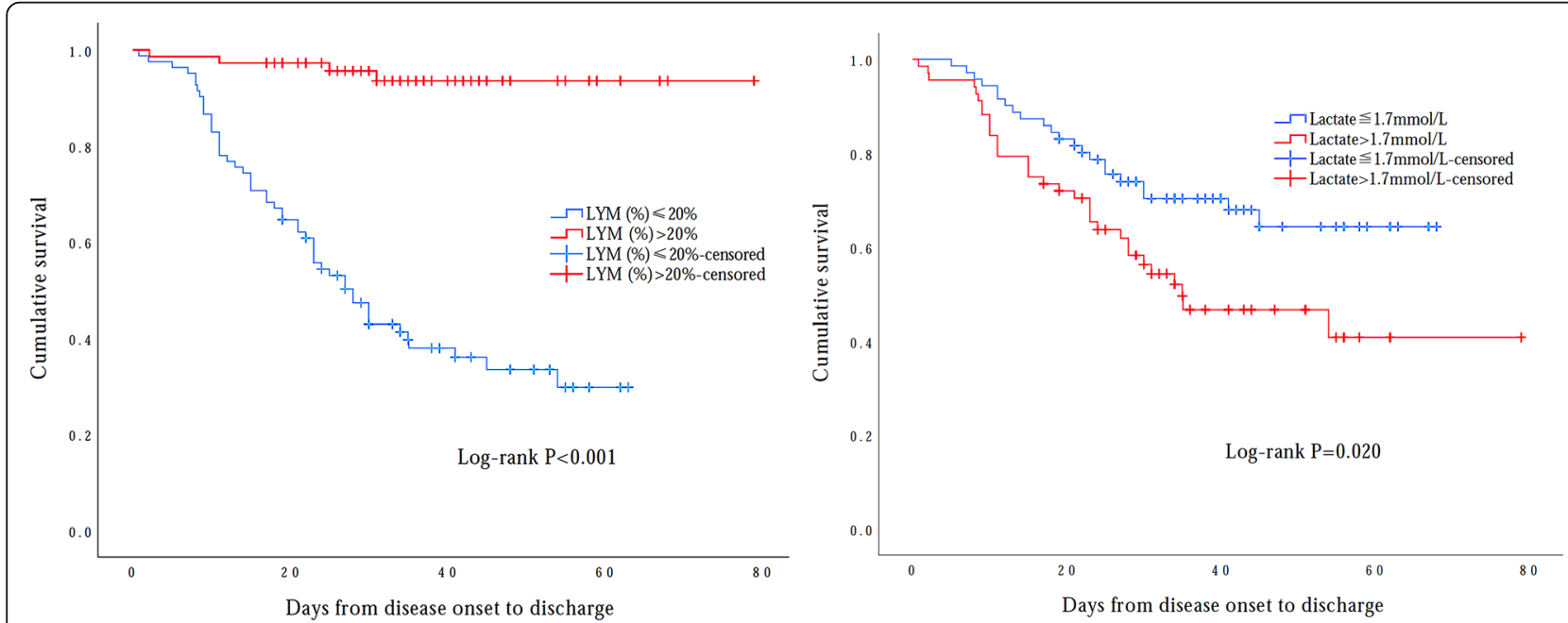

Fig. 1 Kaplan-Meier survival curve for COVID-19 patients with different counts of LYM $(\%)(P<0.001)$ and serum lactate $(P=0.020)(C O V I D-19$, Coronavirus Disease 2019; LYM [\%], Percentage of lymphocytes) 


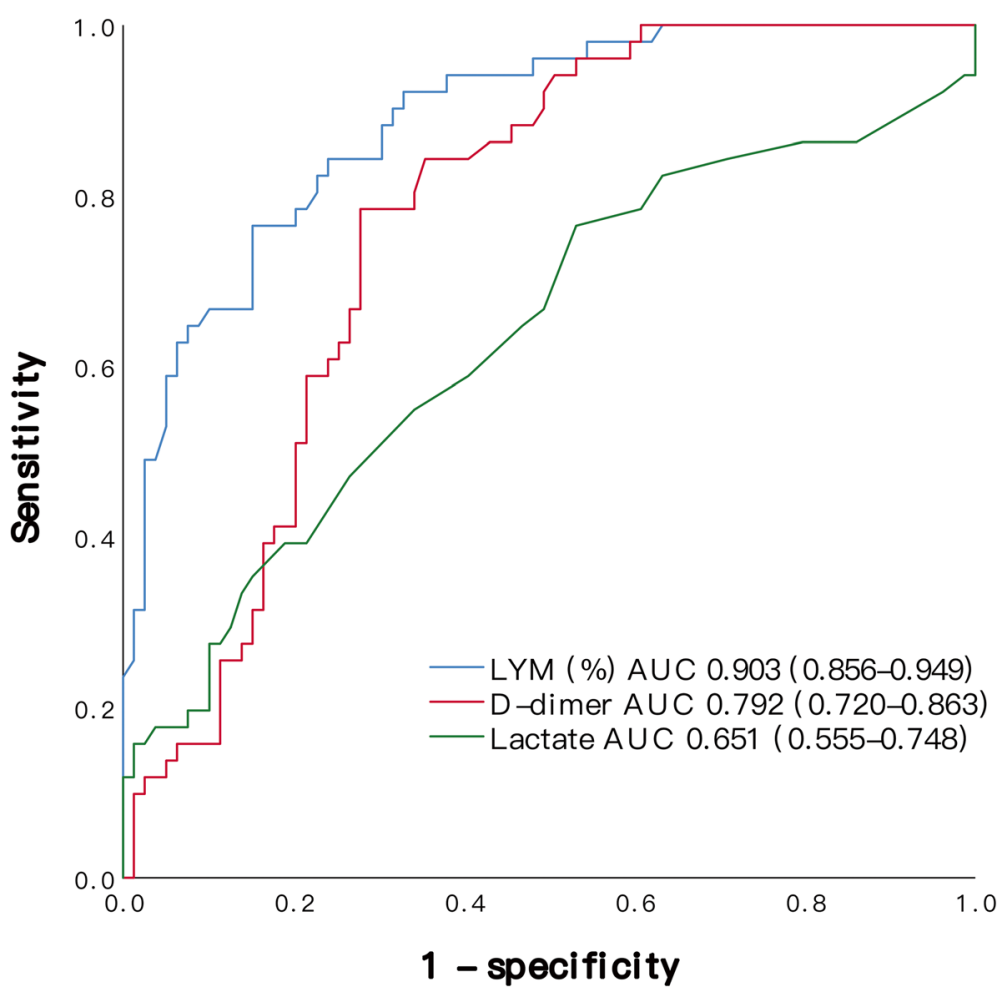

Fig. 2 Receiver-operating characteristic of LYM (\%), D-dimer and lactate upon hospital admission (AUC, Areas under receiver-operating characteristic curve; LYM[\%], Percentage of lymphocytes)

19 patients of advanced ages, especially to whom having multiple comorbidities.

The shorter duration from disease onset to admission was an important factor highly related to odds for death in confirmed cases of COVID-19, which was contrary to previous studies $[21,22]$. This might due to the lack of understanding of this disease in the early pandemic when a large portion of patients who had mild or moderate illness refused to seek proper medical treatment. The shorter time from disease onset to admission for non-survivors in our study might imply a more rapid disease progression than we expected. The etiology of susceptibility to severe lung injury remains unclear. A recent study concluded that the determinants of disease severity seem to stem mostly from host factors, whereas viral genetic variation did not significantly affect outcomes [23]. The balance between angiotensin-converting enzyme (ACE) 1 and ACE 2 activity as the host factors has been implicated in the pathogenesis of respiratory diseases and could play a role in the severity of COVID19 [24].

The multivariable logistic regression assay suggested that the decreased LYM (\%) was an independent risk factor for in-hospital death and further analysis concluded that LYM (\%) was a stronger indicator in predicting in-hospital death by the ROC assay. Previous studies showed that lymphopenia was a risk factor for increased mortality rate for SARS and COVID-19 [14, 25]. While in our study, there was no statistical difference observed in terms of lymphopenia between survivors and nonsurvivors. Liu and colleagues [26] demonstrated that the percentage of lymphocytes (LYM [\%]) was a potential predictor of COVID-19 severity. Considering the fact that the WBC counts were significantly higher in the non-survivors in our cohort which might bias the result, we substituted the absolute lymphocyte count with the LYM [\%] in the regression analysis model and repeated the assay. The decreased LYM (\%) might be explained by the fact that coronavirus was able to destroy lymphocytes during an acute process. The decreased LYM\% may reflect an under-activation and/or over exhausting of the immune system that consequently unable to control COVID-19 infection.

Serum lactate was identified as another risk factor associated with in-hospital death in our study. Lactate has been used as a prognostic marker in predicting the severity and outcome of sepsis and septic shock [27]. Shankar-Hari et al. suggested in their study that the adjusted odds ratio for hospital mortality increased linearly with lactate levels with lactate level $>2 \mathrm{mmol} / \mathrm{L}$ being the cutoff value for the diagnosis of septic shock [28]. This finding had been further confirmed by some 
recent studies [29-31]. In sepsis, patients usually experience hyperlactatemia as a consequence of tissue hypoperfusion, as well as a diminished lactate clearance rate due to dysfunction of the liver and kidney [32]. Inconsistent with our study, Zhou and colleagues [14] identified that sepsis and septic shock was a major complication for COVID-19 patients. Measurement of serum lactate seems to be a simple yet effective strategy to identify patients with increased risks.

A previous study suggested that about $90 \%$ of patients with severe pneumonia had increased coagulation activity, marked by the increased D-dimer concentrations [33]. High levels of D-dimer were proved to be associated with an increased mortality rate in patients with sepsis identified in the emergency room [34]. Previous COVID-19 studies also demonstrated that D-dimer greater than $1 \mu \mathrm{g} / \mathrm{ml}$ was associated with poor prognosis [14]. While in our study, D-dimer was not independently associated with in-hospital death. This discrepancy might be due to the difference in patient selection. Future studies with a larger population are needed to confirm the conclusions.

\section{Limitations}

This study had several limitations. Firstly, this study was a retrospective study conducted in a single-center, with a cohort that might not necessarily representable for the general population. Secondly, by excluding patients still in hospital receiving treatment as of March 20, 2020, the mortality rate in our study might be biased. Finally, the lack of more effective antiviral drugs and life support methods like extracorporeal membrane oxygenation in our hospital might contribute to the poor clinical outcomes in some severe patients.

\section{Conclusions}

Our study indicated that the non-survivors of COVID19 were older and with a disease course that progressed more rapidly compared to survivors. Advanced age, shorter duration from onset to admission, decreased LYM (\%), and increased lactate level upon hospital admission were independent risk factors for in-hospital death of patients with COVID-19 during the early outbreak.

\footnotetext{
Abbreviations

COVID-19: 2019-novel coronavirus; OR: Odds ratio; Cl: Confidence interval: LYM (\%): Percentage of lymphocytes; ARDS: Acute respiratory distress syndrome; ROC: Receiver-operating characteristic; ICU: Intensive care unit; BALF: Bronchoalveolar lavage fluid; KDIGO: Kidney Disease: Improving Global Outcomes; CT: Computed tomography; AUC: Areas under receiver-operating characteristic curve; MERS: Middle east respiratory syndrome; COPD: Chronic obstructive pulmonary disease; WBC: White blood cell; CK: Creatine kinase; LDH: Lactate dehydrogenase; CRP: C-reactive protein; PCT: Procalcitonin; IL6: Interleukin-6; IQR: Interquartile range; NEU (\%): Percentage of neutrophils; ALT: Alanine aminotransferase; AST: Aspartate aminotransferase; BUN: Blood urea nitrogen; CK-MB: Creatine kinase-MB; BNP: Brain natriuretic peptide;
}

PaO2: Partial pressure of oxygen; PaCO2: Partial pressure of carbon dioxide; BE: Base excess; RRT: Renal replacement therapy

\section{Acknowledgements}

We would like to show our respect and gratitude to all the health workers who are at the first line of the outbreak response and fighting against COVID-19.

\section{Authors' contributions}

(I) Conception and design: ZQWand ZGW; (II) Administrative support: ZQW: (III) Provision of study materials or patients: ZQW and ZGW; (IV) Collection and assembly of data: ZQW and ZGW; (V) Data analysis and interpretation: ZGW; (VI) Manuscript writing: ZQW and ZGW; (VII) Final approval of manuscript: ZQW and ZGW.

\section{Funding}

The authors received no internal or external funding from their institution or department.

\section{Availability of data and materials}

The datasets used and/or analysed during the current study are available from the corresponding author on reasonable request.

\section{Ethics approval and consent to participate}

This study was approved by the ethics committee of the Central Hospital of Wuhan. Written informed consent was waived by the ethical commission due to the retrospective nature of the study. Administrative permissions to access the raw data were granted by the Central Hospital of Wuhan ethics committee. The data used in this study was anonymised before its use.

Consent for publication

Not applicable.

\section{Competing interests}

The authors have declared they have no interest.

\section{Author details}

'Department of Cardio-thoracic Surgery, Nanjing Drum Tower Hospital, The Affiliated Hospital of Nanjing University Medical School, Nanjing 210008, China. ${ }^{2}$ Department of Obstetrics and Gynecology, The Central Hospital of Wuhan, Wuhan 430014, China.

Received: 8 July 2020 Accepted: 17 January 2021

Published online: 25 January 2021

\section{References}

1. Wang C, Horby PW, Hayden FG, Gao GF. A novel coronavirus outbreak of global health concern. Lancet. 2020;395(10223):470-473. Epub 2020/01/28. doi: https://doi.org/10.1016/S0140-6736(20)30185-9. PubMed PMID: 31986257.

2. WHO Coronavirus Disease (COVID-19) Dashboard. Available from: https:// covid19.who.int. Accessed 24 October 2020

3. Chan JF, Yuan S, Kok KH, To KK, Chu H, Yang J, et al. A familial cluster of pneumonia associated with the 2019 novel coronavirus indicating personto-person transmission: a study of a family cluster. Lancet. 2020;395(10223): 514-523. Epub 2020/01/28. doi: https://doi.org/10.1016/501406736(20)30154-9. PubMed PMID: 31986261.

4. Jiang Y, Xu J, Zhou C, Wu Z, Zhong S, Liu J, et al. Characterization of cytokine/chemokine profiles of severe acute respiratory syndrome. Am J Respir Crit Care Med 2005;171(8):850-857. Epub 2005/01/20. doi: https://doi. org/10.1164/rccm.200407-857OC. PubMed PMID: 15657466.

5. Ko JH, Park GE, Lee JY, Lee JY, Cho SY, Ha YE, et al. Predictive factors for pneumonia development and progression to respiratory failure in MERSCoV infected patients. J Inf Secur 2016;73(5):468-475. Epub 2016/08/16. doi: https://doi.org/10.1016/j.jinf.2016.08.005. PubMed PMID: 27519621.

6. Huang C, Wang Y, Li X, Ren L, Zhao J, Hu Y, et al. Clinical features of patients infected with 2019 novel coronavirus in Wuhan, China. Lancet 2020;395(10223):497-506. Epub 2020/01/28. doi: https://doi.org/10.1016/ S0140-6736(20)30183-5. PubMed PMID: 31986264.

7. China tNHCo. Interpretation of pneumonia diagnosis and treatment scheme for novel coronavirus infection (trial version 7). Available from: http://www.nhc.gov. 
cn/yzygj/s7652m/202003/a31191442e29474b98bfed5579d5af95.shtml. Accessed 4 Mar 2020.

8. Singer M, Deutschman CS, Seymour CW, Shankar-Hari M, Annane D, Bauer $M$, et al. The Third International Consensus Definitions for Sepsis and Septic Shock (Sepsis-3). JAMA. 2016;315(8):801-810. Epub 2016/02/24. doi: https:// doi.org/10.1001/jama.2016.0287. PubMed PMID: 26903338; PubMed Central PMCID: PMCPMC4968574.

9. Force ADT, Ranieri VM, Rubenfeld GD, Thompson BT, Ferguson ND, Caldwell $E$, et al. Acute respiratory distress syndrome: the Berlin definition. JAMA. 2012;307(23):2526-2533. Epub 2012/07/17. doi: https://doi.org/10.1001/jama. 2012.5669. PubMed PMID: 22797452.

10. Kidney Disease: Improving Global Outcomes(KDIGO) Acute Kidney Injury Work Group. KDIGO Clinical Practice Guideline for Acute Kidney Injury. Kidney Int. 2012;2(Suppl):1-138,

11. Wu F, Zhao S, Yu B, Chen YM, Wang W, Song ZG, et al. A new coronavirus associated with human respiratory disease in China. Nature. 2020;579(7798): 265-269. Epub 2020/02/06. doi: https://doi.org/10.1038/s41586-020-2008-3. PubMed PMID: 32015508; PubMed Central PMCID: PMCPMC7094943.

12. Paules $\mathrm{Cl}$, Marston HD, Fauci AS. Coronavirus Infections-More Than Just the Common Cold. JAMA. 2020. Epub 2020/01/24. doi: https://doi.org/10.1001/ jama.2020.0757. PubMed PMID: 31971553

13. Chen N, Zhou M, Dong X, Qu J, Gong F, Han Y, et al. Epidemiological and clinical characteristics of 99 cases of 2019 novel coronavirus pneumonia in Wuhan, China: a descriptive study. Lancet. 2020;395(10223):507-513. Epub 2020/02/03. doi: https://doi.org/10.1016/S0140-6736(20)30211-7. PubMed PMID: 32007143

14. Zhou F, Yu T, Du R, Fan G, Liu Y, Liu Z, et al. Clinical course and risk factors for mortality of adult inpatients with COVID-19 in Wuhan, China: a retrospective cohort study. Lancet. 2020;395(10229):1054-1062. Epub 2020/03/15. doi: https://doi.org/10.1016/S0140-6736(20)30566-3. PubMed PMID: 32171076.

15. Wang D, Hu B, Hu C, Zhu F, Liu X, Zhang J, et al. Clinical Characteristics of 138 Hospitalized Patients With 2019 Novel Coronavirus-Infected Pneumonia in Wuhan, China. JAMA. 2020. Epub 2020/02/08. doi: https://doi.org/10. 1001/jama.2020.1585. PubMed PMID: 32031570; PubMed Central PMCID: PMCPMC7042881.

16. Li B, Yang J, Zhao F, Zhi L, Wang X, Liu L, et al. Prevalence and impact of cardiovascular metabolic diseases on COVID-19 in China. Clin Res Cardiol 2020. Epub 2020/03/13. doi: https://doi.org/10.1007/s00392-020-01626-9. PubMed PMID: 32161990.

17. Liu M, Liang WN, Chen Q, Xie XQ, Wu J, He X, et al. Risk factors for SARSrelated deaths in 2003, Beijing. Biomed Environ Sci 2006;19(5):336-339. Epub 2006/12/28. PubMed PMID: 17190184

18. Ahmadzadeh J, Mobaraki K, Mousavi SJ, Aghazadeh-Attari J, MirzaAghazadeh-Attari M, Mohebbi I. The risk factors associated with MERS-CoV patient fatality: a global survey. Diagn Microbiol Infect Dis 2020;96(3):114876. Epub 2020/01/22. doi: https://doi.org/10.1016/j.diagmicrobio.2019.114876. PubMed PMID: 31959375.

19. Smits $S L$, de Lang A, van den Brand JM, Leijten $L M$, Wilfred $F$ van lijcken, Eijkemans MJ, et al. Exacerbated innate host response to SARS-CoV in aged non-human primates. PLoS Pathog. 2010;6(2):e1000756. Epub 2010/02/09. doi: https://doi.org/10.1371/journal.ppat.1000756. PubMed PMID: 20140198; PubMed Central PMCID: PMCPMC2816697.

20. Opal SM, Girard TD, Ely EW. The immunopathogenesis of sepsis in elderly patients. Clin Infect Dis 2005;41 Suppl 7:S504-S512. Epub 2005/10/21. doi: https://doi.org/10.1086/432007. PubMed PMID: 16237654.

21. Chen J, Bai H, Liu J, Chen G, Liao Q, Yang J, et al. Distinct clinical characteristics and risk factors for mortality in female COVID-19 inpatients: a sex-stratified large-scale cohort study in Wuhan, China. Clin Infect Dis. 2020. Epub 2020/07/08. doi: https://doi.org/10.1093/cid/ciaa920. PubMed PMID: 32634830; PubMed Central PMCID: PMCPMC7454470.

22. Chen Y, Linli Z, Lei Y, Yang Y, Liu Z, Xia Y, et al. Risk factors for mortality in critically ill patients with COVID-19 in Huanggang, China: A single-center multivariate pattern analysis. J Med Virol. 2020. Epub 2020/10/01. doi: https://doi.org/10.1002/jmv.26572. PubMed PMID: 32997344; PubMed Central PMCID: PMCPMC7537509.

23. Zhang $X$, Tan Y, Ling Y, Lu G, Liu F, Yi Z, et al. Viral and host factors related to the clinical outcome of COVID-19. Nature. 2020;583(7816):437-440. Epub 2020/05/21. doi: https://doi.org/10.1038/s41586-020-2355-0. PubMed PMID: 32434211 .

24. Gomez J, Albaiceta GM, Garcia-Clemente M, Lopez-Larrea C, AmadoRodriguez L, Lopez-Alonso I, et al. Angiotensin-converting enzymes (ACE,
ACE2) gene variants and COVID-19 outcome. Gene. 2020;762:145102. Epub 2020/09/04. doi: https://doi.org/10.1016/j.gene.2020.145102. PubMed PMID: 32882331; PubMed Central PMCID: PMCPMC7456966.

25. Hu X, Deng Y, Wang J, Li H, Li M, Lu Z. Short term outcome and risk factors for mortality in adults with critical severe acute respiratory syndrome (SARS). J Huazhong Univ Sci Technolog Med Sci. 2004;24(5):514-517. Epub 2005/ 01/12. doi: https://doi.org/10.1007/bf02831124. PubMed PMID: 15641708; PubMed Central PMCID: PMCPMC7088722.

26. Liu Y, Yang Y, Zhang C, Huang F, Wang F, Yuan J, et al. Clinical and biochemical indexes from 2019-nCoV infected patients linked to viral loads and lung injury. Sci China Life Sci 2020. Epub 2020/02/13. doi: https://doi. org/10.1007/s11427-020-1643-8. PubMed PMID: 32048163.

27. Oh DH, Kim MH, Jeong WY, Kim YC, Kim EJ, Song JE, et al. Risk factors for mortality in patients with low lactate level and septic shock. J Microbiol Immunol Infect 2019;52(3):418-425. Epub 2017/09/20. doi: https://doi.org/ 10.1016/j.jmii.2017.08.009. PubMed PMID: 28923305.

28. Shankar-Hari M, Phillips GS, Levy ML, Seymour CW, Liu VX, Deutschman CS, et al. Developing a New Definition and Assessing New Clinical Criteria for Septic Shock: For the Third International Consensus Definitions for Sepsis and Septic Shock (Sepsis-3). JAMA. 2016;315(8):775-787. Epub 2016/02/24. doi: https://doi.org/10.1001/jama.2016.0289. PubMed PMID: 26903336; PubMed Central PMCID: PMCPMC4910392.

29. Mikkelsen ME, Miltiades AN, Gaieski DF, Goyal M, Fuchs BD, Shah CV, et al. Serum lactate is associated with mortality in severe sepsis independent of organ failure and shock*. Crit Care Med. 2009;37(5):1670-7. https://doi.org/ 10.1097/CCM.0b013e31819fcf68.

30. Goyal N, Taylor AR, Rivers EP. Relationship between central and peripheral venous oxygen saturation and lactate levels: a prospective study. J Emerg Med 2016;50(6):809-817. Epub 2016/05/24. doi: https://doi.org/10.1016/j. jemermed.2016.03.021. PubMed PMID: 27210904.

31. Chen H, Zhao C, Wei Y, Jin J. Early lactate measurement is associated with better outcomes in septic patients with an elevated serum lactate level. Crit Care. 2019;23(1):351. Epub 2019/11/13. doi: https://doi.org/10.1186/s13054019-2625-0. PubMed PMID: 31711512; PubMed Central PMCID: PMCP MC6849274.

32. Suetrong B, Walley KR. Lactic acidosis in Sepsis: It's not all anaerobic: implications for diagnosis and management. Chest. 2016;149(1):252-261. Epub 2015/09/18. doi: https://doi.org/10.1378/chest.15-1703. PubMed PMID: 26378980.

33. Milbrandt EB, Reade MC, Lee M, Shook SL, Angus DC, Kong L, et al. Prevalence and significance of coagulation abnormalities in communityacquired pneumonia. Mol Med. 2009;15(11-12):438-445. Epub 2009/09/16. doi: https://doi.org/10.2119/molmed.2009.00091. PubMed PMID: 19753144 PubMed Central PMCID: PMCPMC2743205.

34. Rodelo JR, De la Rosa G, Valencia ML, Ospina S, Arango CM, Gomez Cl, et al. D-dimer is a significant prognostic factor in patients with suspected infection and sepsis. Am J Emerg Med 2012;30(9):1991-9. Epub 2012/07/17. doi: https://doi.org/10.1016/j.ajem.2012.04.033. PubMed PMID: 22795996.

\section{Publisher's Note}

Springer Nature remains neutral with regard to jurisdictional claims in published maps and institutional affiliations.

Ready to submit your research? Choose BMC and benefit from:

- fast, convenient online submission

- thorough peer review by experienced researchers in your field

- rapid publication on acceptance

- support for research data, including large and complex data types

- gold Open Access which fosters wider collaboration and increased citations

- maximum visibility for your research: over $100 \mathrm{M}$ website views per year

At $\mathrm{BMC}$, research is always in progress.

Learn more biomedcentral.com/submission 